\title{
Predictor Factors Of Postoperative Pain In Total Hip And Knee Arthroplasties
}

Bárbara Gouveia ${ }^{1}$, Sara Fonseca ${ }^{1}$, Daniela Xará1 ${ }^{1}$, Inês Valdoleiros ${ }^{2}$, Sofia Ferraz ${ }^{1}$, André Sá Rodrigues ${ }^{3}$

1 Centro Hospitalar São João, Porto, 2 Centro Hospitalar do Baixo Vouga, Aveiro, 3 Hospital da Prelada, Porto

\section{BACKGROUND AND GOAL OF STUDY}

Major orthopedic surgeries are associated with postoperative pain (POP) with the total knee (TKA) and total hip (THA) arthroplasties.

\section{MATERIALS AND METHODS}

Prospective cohort study

Population: 95 adult patients admitted to elective THA and TKA, between september and december 2014

Table I- Variables analyzed in the pre and postoperative questionnaires

\begin{tabular}{|c|c|}
\hline Preoperative questionnaire & $\begin{array}{l}\text { Postoperative } \\
\text { questionnaire }\end{array}$ \\
\hline - Demographic variables & \multirow{8}{*}{$\begin{array}{l}\text { - } \quad \text { Intensity of POP } \\
\text { (Brief Pain Inventory) } \\
-\quad \text { Minimum } \\
-\quad \text { Average } \\
-\quad \text { Maximum } \\
-\quad \text { At the time }\end{array}$} \\
\hline - Type of arthroplasty & \\
\hline - ASA physical status & \\
\hline - Body Mass Index (BMI) & \\
\hline - Preoperative chronic pain & \\
\hline - Depression diagnosis & \\
\hline - Depressive profile & \\
\hline - Medication for depression & \\
\hline
\end{tabular}

Descriptive analysis of variables was performed and non-parametric tests were used in the analysis of POP levels. It was performed a multiple linear regression to determine the significant predictors of the POP.

\section{RESULTS}

We included 95 patients, all caucasian, 58 of which underwent TKA and 37 to THA.

Table II - POP according to sex, age, BMI, ASA physical status, type of arthroplasty, depression diagnosis, depression medications, preoperative chronic pain

\begin{tabular}{|c|c|c|c|c|c|c|c|c|c|}
\hline & & $\begin{array}{l}\text { Minimal } \\
\text { POP }\end{array}$ & $p$ & $\begin{array}{c}\text { Average } \\
\text { POP }\end{array}$ & $\mathrm{p}$ & $\begin{array}{l}\text { Maximum } \\
\text { POP }\end{array}$ & $\mathrm{p}$ & $\begin{array}{l}\text { At the time } \\
\text { POP }\end{array}$ & $p$ \\
\hline \multirow{2}{*}{ Sex } & Female & $1,47+-1,77(0-6)$ & \multirow{2}{*}{$\begin{array}{l}\hat{A} \\
\text { O }\end{array}$} & $4,84+-1,59(0-8)$ & \multirow{2}{*}{ ถู่ } & $\begin{array}{c}6,97+-2,19(3- \\
10)\end{array}$ & \multirow{2}{*}{$\begin{array}{l}\text { 草 } \\
0 \\
0 \\
0\end{array}$} & $2,49+-2,71(0-9)$ & \multirow{2}{*}{$\stackrel{\substack{\infty \\
N \\
0}}{0}$} \\
\hline & Male & $1,15+-1,26(0-4)$ & & $4,04+-1,81(1-8)$ & & $\begin{array}{c}5,74+-1,87(3- \\
10)\end{array}$ & & $3,19+-2,59(0-7)$ & \\
\hline \multirow{2}{*}{ Age } & $<65$ & $1,24+-1,58(0-6)$ & \multirow{2}{*}{$\begin{array}{l}9 \\
\substack{9 \\
0 \\
0}\end{array}$} & $4,46+-1,53(0-8)$ & \multirow{2}{*}{$\underset{b}{b}$} & $\begin{array}{c}6,44+-2,01(3- \\
10)\end{array}$ & \multirow{2}{*}{ 离 } & $2,53+-2,83(0-8)$ & \multirow{2}{*}{ 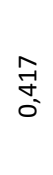 } \\
\hline & $\geqq 65$ & $1,56+-1,73(0-6)$ & & $4,8+-1,87(1-8)$ & & $\begin{array}{l}6,85+-2,36(3- \\
10)\end{array}$ & & $2,88+-2,5)(0-9)$ & \\
\hline \multirow{5}{*}{ BMI } & $\leqq 18$ & $0,5+-0,71(0-1)$ & \multirow{5}{*}{$\begin{array}{l}\infty \\
0 \\
0 \\
0\end{array}$} & $4,5+-3,54(2-7)$ & \multirow{5}{*}{$\begin{array}{c}\text { N} \\
0 \\
0\end{array}$} & $6,5+-4,95(3-10)$ & \multirow{5}{*}{ 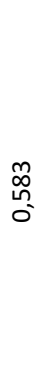 } & $1,5+-2,12(0-3)$ & \multirow{5}{*}{ 总 } \\
\hline & $>18$ and $<25$ & $0,67+-1,11(0-3)$ & & $4,13+-1,3(2-6)$ & & $6+-2,14(3-9)$ & & $3,53+-2,88(0-8)$ & \\
\hline & $\geqq 25$ and $<30$ & $1,28+-1,32(0-4)$ & & $4,58+-1,69(0-8)$ & & $\begin{array}{c}6,58+-2,05(3- \\
10)\end{array}$ & & $2,47+-2,43(0-7)$ & \\
\hline & $\geqq 30$ and $<35$ & $1,52+-1,93(0-6)$ & & $4,85+-1,66(2-8)$ & & $\begin{array}{c}7,15+-2,21(3- \\
10)\end{array}$ & & $2,42+-3,05(0-9)$ & \\
\hline & $\geqq 35$ & $3+-2,27(0-6)$ & & $4,88+-2,17(2.8)$ & & $\begin{array}{c}6,25+-2,19(3- \\
10)\end{array}$ & & $3,38+-2,56(0-7)$ & \\
\hline \multirow{4}{*}{$\begin{array}{c}\text { ASA } \\
\text { physical } \\
\text { status }\end{array}$} & 1 & $1+-1(0-2)$ & \multirow{4}{*}{$\begin{array}{l}\text { 总 } \\
\text { ô. }\end{array}$} & $4,67+-3,06(2-8)$ & \multirow{4}{*}{ 总 } & $6+-3,61(3-10)$ & \multirow{4}{*}{$\begin{array}{c}\vec{m} \\
0 \\
0\end{array}$} & $2+-3,46(0-6)$ & \multirow{4}{*}{ 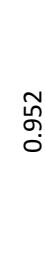 } \\
\hline & 2 & $1,31+-1,62(0-6)$ & & $\begin{array}{c}4,52+-1,61(0- \\
8)\end{array}$ & & $\begin{array}{c}6,49+-2,11(3- \\
10)\end{array}$ & & $2,72+-2,7(0-9)$ & \\
\hline & 3 & $2+-1,96(0-6)$ & & $5,38+-1,89(1-8)$ & & $8+-1,91(4-10)$ & & $2,77+-2,83(0-7)$ & \\
\hline & 4 & $1+-0(1-1)$ & & $4+-0(4-4)$ & & $6+-0(6-6)$ & & $3+-0(3-3)$ & \\
\hline \multirow{2}{*}{$\begin{array}{c}\text { Type of } \\
\text { arthroplasty }\end{array}$} & THA & $0,89+-1,17(0-4)$ & \multirow{2}{*}{ : } & $3,92+-1,85(0-8)$ & \multirow{2}{*}{ : } & $5,84+-2,2(3-10)$ & \multirow{2}{*}{ 望 } & $2,84+-2,76(0-8)$ & \multirow[b]{2}{*}{0} \\
\hline & TKA & $1,69+-1,83(0-6)$ & & $5,05+-1,42(2-8)$ & & $7,12+-2(3-10)$ & & $2,58+-2,65(0-9)$ & \\
\hline \multirow{2}{*}{$\begin{array}{c}\text { Depression } \\
\text { diagnosis }\end{array}$} & No & $1,41+-1,6(0-6)$ & \multirow{2}{*}{ 嵑 } & $4,37+-1,81(0-8)$ & \multirow{2}{*}{ : } & $6,1+-1,94(3-10)$ & \multirow{2}{*}{ 商 } & $3,14+-2,71(0-8)$ & \multirow{2}{*}{ 委 } \\
\hline & Yes & $1,33+-1,74(0-6)$ & & $5+-1,39(2-8)$ & & $\begin{array}{c}7,47+-2,27(3- \\
10)\end{array}$ & & $1,94+-2,5(0-9)$ & \\
\hline \multirow{2}{*}{$\begin{array}{l}\text { Depression } \\
\text { medication }\end{array}$} & No & $0,67+-0,82(0-2)$ & \multirow{2}{*}{ 总 } & $5,17+-1,17(4-7)$ & \multirow{2}{*}{ 答 } & $8+-2,76(3-10)$ & $\hat{m}$ & $1+-1,26(0-3)$ & $n$ \\
\hline & Yes & $1,47+-1,85(0-6)$ & & $4,97+-1,45(2-8)$ & & $7,37+-2,2(3-10)$ & 0 & $2,13+-2,65(0-9)$ & 0 \\
\hline & No & $0,92+-1,5(0-6)$ & $\stackrel{\infty}{\stackrel{\infty}{2}}$ & $3,84+-1,62(2-8)$ & 列 & $\begin{array}{c}5,92+-2,31(3- \\
10)\end{array}$ & n & $1,5+-2,43(0-7)$ & 皮 \\
\hline & Yes & $34+-1,67(0-6)$ & & $4,89+-1,63(0-8)$ & & $\begin{array}{c}6,87+-2,07(3- \\
10)\end{array}$ & & $3,09+-2,66(0-9)$ & \\
\hline
\end{tabular}

Table III- POP according to the type of arthroplasty by sex and age

\begin{tabular}{|c|c|c|c|c|c|c|c|c|c|}
\hline & & $\begin{array}{c}\text { Minimal } \\
\text { POP }\end{array}$ & p & $\begin{array}{c}\text { Average } \\
\text { POP }\end{array}$ & $p$ & $\begin{array}{c}\text { Maximum } \\
\text { POP }\end{array}$ & p & $\begin{array}{c}\text { At the Time } \\
\text { POP }\end{array}$ & $p$ \\
\hline \multirow{2}{*}{ Female } & THA & 7 & 0,159 & $4,1 \pm 1,79$ & $0,025^{*}$ & $6,1 \pm 2,36$ & $0,033^{*}$ & $2,67 \pm 2,9$ & 0,777 \\
\hline & TKA & $1,72 \pm 1,93$ & & $5,17 \pm 1,39$ & & $7,36 \pm 2$ & & $2,4 \pm 2,64$ & \\
\hline \multirow{2}{*}{ Male } & THA & $0,88 \pm 1,15$ & 0,167 & $3,69 \pm 1,96$ & 0,152 & $5,5 \pm 2$ & 0,321 & $3,06 \pm 2,64$ & 0,776 \\
\hline & TKA & $1,55 \pm 1,37$ & & $4,55 \pm 1,51$ & & $6,09 \pm 1,7$ & & $3,4 \pm 2,63$ & \\
\hline \multirow{2}{*}{$<65$} & THA & $0,87 \pm 1,14$ & 0,258 & $3,87 \pm 1,66$ & $0,029^{*}$ & $5,78 \pm 2,02$ & $0,032^{*}$ & $2,83 \pm 2,89$ & 0,583 \\
\hline & TKA & $1,52 \pm 1,81$ & & $4,9 \pm 1,27$ & & $6,94 \pm 1,88$ & & $2,3 \pm 2,81$ & \\
\hline \multirow[t]{2}{*}{$\geqq 65$} & THA & $0,93 \pm 1,27$ & 0,122 & $4 \pm 2,18$ & 0,052 & $5,93 \pm 2,56$ & 0,082 & $2,86 \pm 2,66$ & 0,989 \\
\hline & TKA & $1,89 \pm 1,87$ & & $5,22 \pm 1,58$ & & $7,33 \pm 2,15$ & & $2,89 \pm 2,47$ & \\
\hline
\end{tabular}

Table IV- Multiple linear regression

\begin{tabular}{|c|c|c|c|c|c|c|c|c|}
\hline & \multicolumn{2}{|l|}{$\begin{array}{c}\text { Minimal } \\
\text { POP }\end{array}$} & \multicolumn{2}{|l|}{$\begin{array}{l}\text { Average } \\
\text { POP }\end{array}$} & \multicolumn{2}{|c|}{$\begin{array}{l}\text { Maximum } \\
\text { POP }\end{array}$} & \multicolumn{2}{|c|}{$\begin{array}{l}\text { At the Time } \\
\text { POP }\end{array}$} \\
\hline & B (IC 95\%) & $D$ & $\mathrm{~B}(\mathrm{IC} 95 \%)$ & $D$ & B (IC 95\%) & D & B (IC 95\%) & D \\
\hline BMI $\leqq 18$ & & & $-2,64(-5,24 / 10,04)$ & $0,047^{*}$ & & & & \\
\hline BMI $>18$ and $<25$ & & & $-2,22(-3,66 / 10,77)$ & $0,003^{*}$ & & & & \\
\hline BMI $\geqq 25$ and $<30$ & & & $-1,56(-2,86 / 10,25)$ & $0,020^{*}$ & & & & \\
\hline BMI $\geqq 30$ and $<35$ & & & $-1,13(-2,49 / 0,23)$ & 0,103 & & & & \\
\hline Depression & $0,08(-0,69 / 0,84)$ & 0.847 & & & $-0,71(-1,69 / 0,28)$ & 0.156 & $-1,57(0,4612,69)$ & $0,006^{*}$ \\
\hline $\begin{array}{l}\text { Type of Surgery - } \\
\text { THA }\end{array}$ & $-0,84(-1,533 / 0,15)$ & 0,018 & & & $-0,67(-1,5910,26)$ & 0,156 & & \\
\hline Chronic Pain & $0,55(-0,2111,32)$ & 0,155 & $0,31(-0,4711,09)$ & 0,429 & $0,10(-0,901,1,10)$ & 0,848 & $1,71(-2,96 / 10,47)$ & $0,008^{\circ}$ \\
\hline$R^{2}$ & 0,280 & & 0.210 & & 0,385 & & 0,201 & \\
\hline
\end{tabular}

\section{DISCUSSION AND CONCLUSION}

Some predictive factors of POP are described after orthopedic surgery, but the most consistently identified are: age, type of surgery, preoperative pain, anxiety and depression ${ }^{2,3}$.

In this study, women and younger patients undergoing TKA had higher levels of POP, however, age and gender were not identified as predictors of POP and physical status ASA didn't interfere with POP intensity. We also found an association between the presence of chronic pain in the preoperative period and the development of more intense POP. Type of surgery, BMI, presence of chronic pain and absence of depression were identified as pain predictors after TKA and THA.

The identification of these predictors contributes to the individualized optimization of analgesic regimens, with a consequent improvement in postoperative pain recovery after THA and TKA. 